\title{
Philosophiques
}

\section{Leroux, J., La Sémantique des théories physiques, collection Philosophica, Ottawa, Les Presses de l'Université d'Ottawa,} 1988.

\section{Serge Robert}

Volume 16, numéro 1, printemps 1989

URI : https://id.erudit.org/iderudit/027078ar

DOI : https://doi.org/10.7202/027078ar

Aller au sommaire du numéro

Éditeur(s)

Société de philosophie du Québec

\section{ISSN}

0316-2923 (imprimé)

1492-1391 (numérique)

Découvrir la revue

Citer ce compte rendu

Robert, S. (1989). Compte rendu de [Leroux, J., La Sémantique des théories

physiques, collection Philosophica, Ottawa, Les Presses de l'Université

d'Ottawa, 1988.] Philosophiques, 16(1), 230-234.

https://doi.org/10.7202/027078ar d'utilisation que vous pouvez consulter en ligne.

https://apropos.erudit.org/fr/usagers/politique-dutilisation/ 
LEROUX, J., La Sémantique des théories physiques, collection Philosophica, Ottawa, Les Presses de l'Université d'Ottawa, 1988.

\section{par Serge Robert}

L'auteur commence par nous rappeler comment la distinction qu'avait faite Reichenbach (1938) entre contexte de découverte et contexte de justification a orienté la philosophie des sciences dans une perspective justificationniste (p. 2), en laissant le problème de la découverte scientifique 
aux historiens et aux psychologues. Le justificationnisme philosophique s'est plus particulièrement exprimé comme théorie de la démarcation entre science et non-science, par exemple dans une théorie du sujet (comme la théorie de la rationalité chez Popper) ou dans une théorie de l'objet (comme la théorie de l'empiricité chez Carnap) (p. 3). L'auteur considère que de chercher ainsi un fondement démarcationniste à la science, en la comparant à ce qu'elle n'est pas, est une approche externe à la théorie scientifique; il préfère plutôt tenter de fonder la théorie de l'intérieur, en opposant une approche fondationnaliste à cette perspective démarcationniste. Il définit ainsi son projet comme étant centré sur la notion de théoricité, qu'il juge plus fondamentale que celle de scientificité (p. 5). La question centrale devient alors la suivante : que faisons-nous quand nous théorisons mathématiquement sur l'expérience, comme c'est le cas dans la physique mathématique ? Cette approche fondationnelle devrait, au dire de l'auteur, dépasser la dualité épistémologique traditionnelle du sujet et de l'objet, dans la mesure où la science physique comprend déjà en elle-même sa propre théorie du sujet (théorie de l'observateurr) et sa théorie de l'objet (théorie de la mesure) (p. 7).

L'ouvrage constitue plus particulièrement une analyse fondationnelle de la théorie physique, laquelle se distingue des autres sciences par le fait que " les mathématiques y interviennent au niveau même de la conceptualisation " (p. 8) et qu'elle forme ainsi une métrisation de l'expérience, un ensemble d'hypothèses «sur la réalisation physique (empirique) de certaines structures mathématiques» (p. 8). L'auteur aborde plus spécifiquement la théorie physique sous trois angles: sa structure mathématique, son interprétation physique et son domaine d'application (p. 7). Trois approches fondationnelles sont successivement étudiées : l'empirisme logique (Carnap, Hempel,...), le structuralisme (Suppes, Sneed,...) et le constructivisme (Gauthier).

En campant le contexte historique de la tradition analytique en philosophie de la physique, l'auteur résume bien les thèses de l'empirisme logique qui fondent la received view : la théorie scientifique est l'ensemble des conséquences logiques d'un système d'axiomes (formalisme syntaxique) et son interprétation est directe et fixe (p. 13). La théorie est donc vue comme un ensemble d'énoncés (statement view) qui peut être interprété par observation directe, dans un langage observationnel neutre directement relié à cette observation. L'interprétation de la théorie se fait alors dans la perspective de la théorie des modèles héritée de Tarski : on a ainsi une sémantique extensionnelle qui se fonde sur une ontologie ensembliste (p. 81).

Leroux nous fait alors parcourir l'évolution de la théorie empiriste logique de l'interprétation sémantique des théories physiques. On débute avec Carnap, qui avait d'abord utilisé le concept de définition explicite, selon lequel les prédicats théoriques peuvent être considérés comme équivalant à des prédicats du langage observationnel (p. 22). Les définitions explicites avaient l'avantage d'assurer la non-créativité sémantique et l'unicité d'interprétation des théories (p. 23), tout en les fondant empiriquement. Mais ces 
définitions se sont cependant vite avérées trop contraignantes. Carnap essaya alors la notion de définition conditionnelle, où l'équivalence du prédicat théorique avec un prédicat du langage observationnel ne vaut que lorsque certaines conditions expérimentales (cf. les termes dispositionnels) sont rencontrées (p. 24). Ensuite, la notion encore plus lâche de définition partielle a remplacé celle de définition conditionnelle, de façon à ne plus garantir la non-créativité sémantique (p. 25).

Cet échec de la réductibilité des théories physiques à l'observation a amené Carnap à ce qui sera par la suite considéré comme le modèle empiriste standard, où le langage théorique et le langage observationnel sont médiatisés par des règles de correspondance (pp. 26-7). Mais les règles de correspondance ont elle aussi posé des problèmes. C'est ainsi que le réalisme sémantique présupposé par l'empirisme logique a ouvert le porte à un certain conventionnalisme (p. 71) et que, dans le sens du holisme épistémologique quinien, la distinction radicale des énoncés analytiques a priori et des énoncés synthétiques a posteriori, ou encore la distinction des axiomes théoriques et des règles de correspondances, a été atténuée. Dans la même foulée de remise en question de l'empirisme logique, les Kuhn et Feyerabend ont questionné la neutralité du langage observationnel, considérant ce dernier comme chargé de théorie (theory-ladenness) (p. 80), et ont insisté sur la variation sémantique (meaning variance) des termes théoriques (p. 85). Dès lors, la dualité du théorique et de l'observable ne pouvait plus tenir (p. 73).

C'est dans ce contexte d'incapacité à démarquer nettement le théorique de l'observationnel, de prégnance de la théorie sur l'observation, de variance sémantique et d'échec des règles de correspondance, que va naître l'approche structuraliste de Sneed (p. 86-7). La trichotomie théorie formelle-règles de correspondance-interprétation physique est remplacée par une dichotomie structures formelles-applications empiriques (p. 88). C'est donc dire que la théorie physique n'est plus considérée comme un ensemble d'énoncés axiomatisés (statement view), mais comme ayant déjà en elle-même des structures ensemblistes définies sur le langage de la théorie (p. 89). De plus, on remplace la notion d'interprétation physique des énoncés de la théorie par celle d'applications visées des structures, de façon à remplacer le problème sémantique de la vérité empirique des énoncés par la notion instrumentaliste de réussite d'application des structures à des domaines d'objets (p. 90). L'approche de Sneed s'appuie ainsi sur un pragmatisme d'inspiration kuhnienne, où la théorie est jugée selon l'usage qu'en font les scientifiques pour manipuler les objets. Selon ce point de vue, la théorie comprend un noyau et des déploiements spécialisés: l'expansion du noyau et l'augmentation du domaine visé d'applications rendent compte du progrès empirique de la science normale, en respectant la thèse Duhem-Quine sur la relation holiste de la théorie à l'expérience, comme le développement de spécialisations du noyau correspond au progrès théorique de la science normale (p. 104).

L'ouvrage s'achève sur une comparaison entre la théorie constructiviste d'Y. Gauthier et les deux approches précédentes. On y rappelle comment 
l'antiréalisme de Gauthier privilégie le syntaxique contre le sémantique, en insistant sur la surdétermination du physique par le mathématique (p. 135), dans une conception de la théorie physique où le formalisme logico-mathématique est interprété par homomorphisme dans une théorie physique, le tout étant à nouveau interprété par homomorphisme dans des données physiques fondamentales fortement chargées de théorie (p. 131-2).

L'ouvrage traite avec justesse de chacune des trois théories fondationnelles et identifie bien les faiblesses des deux premières, principalement en ce qui concerne comment elles se situent relativement au débat épistémologique entre le réalisme et l'instrumentalisme. Ainsi, Leroux nous montre bien qu'en utilisant la théorie des modèles, l'empirisme logique tente de fonder la théorie physique d'une manière réaliste, mais que ses difficultés d'ajuster la théorie sur l'empirie, par exemple avec les règles de correspondance, le font pencher vers un certain instrumentalisme. Mais l'instrumentalisme ne suffit pas à l'empirisme logique qui n'abandonne pas l'idée d'une adéquation empirique de la théorie (PP. 69-71). L'empirisme logique est donc tiraillé, sans pouvoir trancher, entre le réalisme et l'instrumentalisme. Quant au structuralisme, sa perspective pragmatiste le rend nettement instrumentaliste et antiréaliste. Mais Leroux critique le concept instrumentaliste d'application qu'on y retrouve, en nous disant que: «Le problème majeur de cette approche est que le domaine d'application semble être déterminé de façon purement à priori, alors que dans le cas des théories physiques, le domaine d'application est déterminé progressivement au fil des investigations empiriques» (p. 93). C'est un peu dans le même ordre d'idées que Sneed a donné à son concept d'application des accents réalistes qui le tamènent dans un lieu épistémologique plus voisin de l'empirisme logique qu'on aurait pu le soupçonner (p. 115). Le structuralisme n'échapperait donc pas, lui non plus, à ce tiraillement entre réalisme et instrumentalisme.

C'est en voulant éviter l'engagement ontologique qu'exige le réalisme et en refusant l'instrumentalisme comme étant une manière d'éviter le problème épistémologique du mode d'ancrage de la théorie dans l'empirie, que Leroux rermine son ouvrage en faisant appel au constructivisme. Ce qui est ici regrettable c'est que l'ouvrage ne traite que sommairement de l'approche de Gauthier et qu'il nous apparaît difficile de voir jusqu'où l'auteur est prêt à le suivre et à partir d'où il s'en démarque.

Leroux insiste sur l'importance de rendre compte de l'empirie par la théorie scientifique, et comme on sait par ailleurs que Gauthier considère l'expérience empirique comme fortement construite, on s'étonne que l'auteur ne traite pas de l'empirisme constructif de van Fraassen : le semi-constructivisme de ce dernier (The Scientific Image), dont moi-même je me rapproche, ne conviendrait-il pas mieux aux préoccupations de Leroux?

En suivant Reichenbach dans son exclusion du contexte de découverte hors du champ de la philosophie des sciences, sans revenir sur la question, l'auteur semble négliger le fait que c'est la réintroduction de ce contexte chez ceux qu'on a appelé, à la suite de Hanson et Kuhn, the friends of discovery, 
que l'on a pu découvrir la variance sémantique des théories et la prégnance de la théorie sur l'observation, et que des alternatives à l'empirisme logique, comme le structuralisme, ont pu apparaître. Dans le même ordre d'idées, quand Leroux soutient que Kuhn n'a pas été le moteur de la théorie structuraliste, s'il veut dire que Kuhn n'y a pas directement contribué, alors j'en conviens. Mais il ne faudrait pas oublier que la théorie Kuhnienne des paradigmes est cependant la conception de la science qui a sûrement le plus marqué les structuralistes et dont la théorie a voulu rendre compre.

Quant à la distinction qui est fait entre le fondationnalisme et le démarcationnisme, bien qu'elle paraisse acceptable à première vue, l'analyse la montre beaucoup plus relative qu'il ne semble : en effet, en s'attaquant au rapport privilégié que la théorie scientifique entretient avec l'expérience, le fondationnalisme se trouve à démarquer indirectement la science des discours non scientifiques et, inversement, en tentant de fonder la scientificité de la science, la théorie démarcationniste fonde les rapports de la théorie scientifique à l'expérience. C'est pourquoi l'auteur se prive d'un pan important de la philosophie des sciences quand il tient à cette opposition un peu artificielle entre fondationnalisme et démarcationnisme.

En se rapprochant du constructivisme, Leroux fait allusion à la philosophie frégéenne du langage, où un passage par le sens est nécessaire à l'assignation de la référence, mais en associant le sens à des procédures de vérification (p. 137). On aurait aimé savoir ici pourquoi l'auteur ne va pas plus loin dans cette direction : j'aurais aimé qu'il nous expose, soit les raisons d'une aversion pour l'intensionnalisme, soit un traitement de la constitution de la théorie scientifique par le recours à l'intentionnalité.

En résumé, l'ouvrage est une belle présentation de quelques philosophies de la physique qui ont particulièrement marqué les dernières décennies. $A$ ce strict niveau, on a affaire à ce qui pourrait constituer un bon manuel de référence pour un cours d'introduction à la philosophie de la physique. Le texte va cependant plus loin par sa perspective critique et sa volonté de contribuer au débat. Bien que je partage plusieurs des critiques formulées par l'auteur, c'est ici cependant que l'on reste un peu sur son appétit. Refusant l'instrumentalisme et un certain réalisme dur, privilégiant l'extensionnalisme en se taisant sur l'intensionnalisme, acceptant les thèses de la variance sémantique et de la prégnace de la théorie sur l'observation, se ralliant au constructivisme en voulant maintenir un empirisme et un réalisme minimaux, Leroux n'aboutit pas sur une position claire. Il faut dire sur ce que le problème abordé est extrêmement complexe, que nous sommes devant un premier ouvrage et que l'auteur connaît suffisamment la question pour être prudent. J'ai néanmoins fortement l'impression que Leroux trouvera sa voie entre les différentes tendances à travers lesquelles il chemine. C'est pourquoi j'apprécie sa performance actuelle, mais je suis surtout dans l'attente du prochain ouvrage qu'il produira sur la question.

Département de philosophie

Université du Québec à Montréal 